\title{
Coherent Electron Interference of a Split HOLZ Line from a Strained Silicon Crystal
}

Rodney A. Herring ${ }^{1}$, Koh Saitoh ${ }^{2}$, Nobuo Tanaka ${ }^{2}$ and Takayoshi Tanji ${ }^{2}$

${ }^{1}$ CAMTEC, MENG, University of Victoria, British Columbia V8N 4T6 Canada

${ }^{2}$ EcoTopia, Nagoya University, Nagoya, 461-8603 Japan

Recent methods of k-space interference by means of an electron biprism on the in-focus diffraction plane have revealed that practically all electron intensities, elastic and inelastic, originating from a crystal or amorphous material generated by a coherent electron source of a TEM have sufficient coherence to form fringes when self-interfered under a wide range of diffraction conditions [1, 2]. Here we report the formation of fringes giving phase information of HOLZ lines by the self-interference of a split higher-order Laue zone (HOLZ) reflection line observed in a convergent-beam electron diffraction (CBED) disc produced from a Si substrate material located near a strained $\mathrm{Si}_{0.7} \mathrm{Ge}_{0.3} / \mathrm{Si}$ epilayer grown by molecular beam epitaxy. This method of electron interference on the diffraction plane, often referred to as Diffracted Beam Interferometry (DBI), Fig. 1, compliments a recently developed method of CBED [3] used to determine the lattice parameters of strained $\mathrm{Si}$, as well as, the parameters characterizing the bending strain of its lattice and the direction and magnitude of the displacement field of the bending strain such that twodimensional maps of the lattice parameter, the direction and the relative magnitude of the displacement field could be produced by fitting two Hough transforms of experimental and kinematically simulated HOLZ line patterns.

An undetermined split HOLZ line within the 000 disc located close to the [320] zone axis was used for this experiment, Fig. 2a. Experimental details include using a JEOL JEM-2010F operated at $200 \mathrm{kV}$, a probe of $\sim 5 \mathrm{~nm}$, specimen thickness of $\sim 200 \mathrm{~nm}$, energy filtered electrons using a GIF Tridiem and the zero loss electrons having a $5 \mathrm{eV}$ window. Using the same split HOLZ line in a dark-field CBED beam and placing the electron biprism parallel to the split HOLZ line, a positive voltage was applied to the biprism resulting in the intensities of the split HOLZ line converging for their selfinterfered. The interference fringes run parallel to the line direction of the split HOLZ line (Fig. $2 \mathrm{~b}-2 \mathrm{~d}$ ). Increasing the biprism voltage from $0 \mathrm{~V}$ to $\sim 30 \mathrm{~V}$ decreased the interference width and increased the spatial frequency of the fringes. Fig 3 shows more clearly the fine fringes in Fig. 2d revealing phase shifts existing at the intersections with other HOLZ lines that pass through the split HOLZ line.

Although the full extent of the information provided by this method of interference is not yet known, these experiments reveal, as expected, that the CBED intensity has good coherence by the high contrast fringes produced, which may enable a better 2D measurement of the crystal strain. As well, using this method to measure multiple phase shifts of the split HOLZ lines may enable the determination of the 3D displacement vector of the strained lattice.

1. R.A. Herring, “Electron Beam Coherence Measurements using Diffracted Beam Interferometry /Holography" J. Electron Microscopy 58(3) (2009) 213-221. 2. R.A. Herring, K. Saitoh, N. Tanaka, and T. Tanji, "Coherent Electron Interference from Amorphous TEM Specimens," J. Electron Microscopy 59(5) (2010) 1-9. 3. K. Saitoh, Y. Yasuda, M. Hamabe and N. Tanaka, "Automated characterization of bending and expansion of a lattice of a Si substrate near a SiGe/Si interface by using split HOLZ line patterns" J. Electron Microscopy (2010) 1-12. 


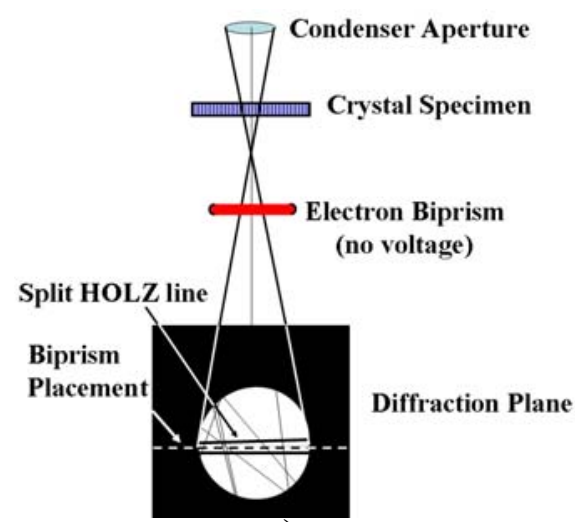

a)

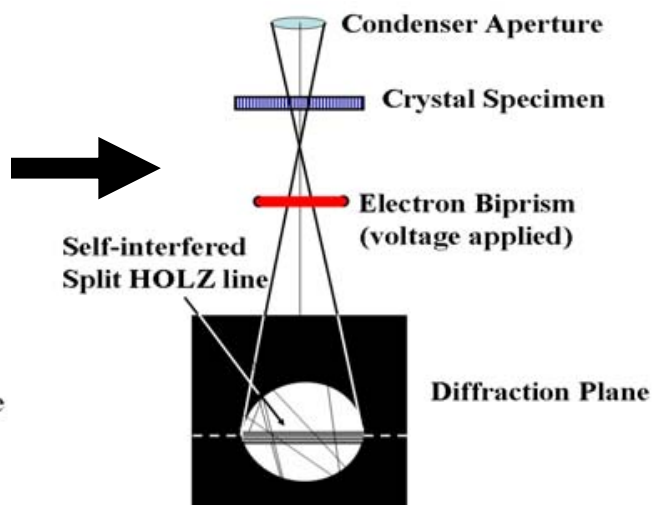

b)

Fig 1 - Schematic of the DBI interference of a split HOLZ line showing placement of biprism between split HOLZ line before a) and after b) the voltage is applied.
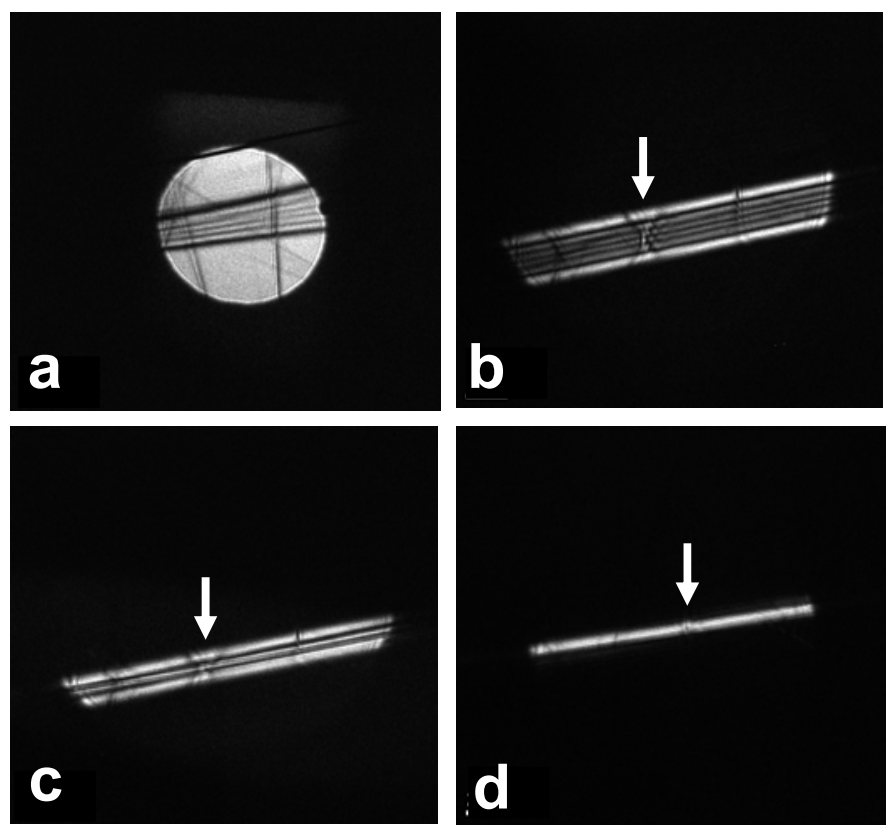

Fig 2 - Experimental images showing in a) a split HOLZ line running horizontally through the 000 disc, b) the same split HOLZ line running through a diffraction disc, c) self-interference of the split HOLZ line by the biprism and d) same as c) but increased biprism voltage. White arrow indicates same intersection point of another HOLZ line.

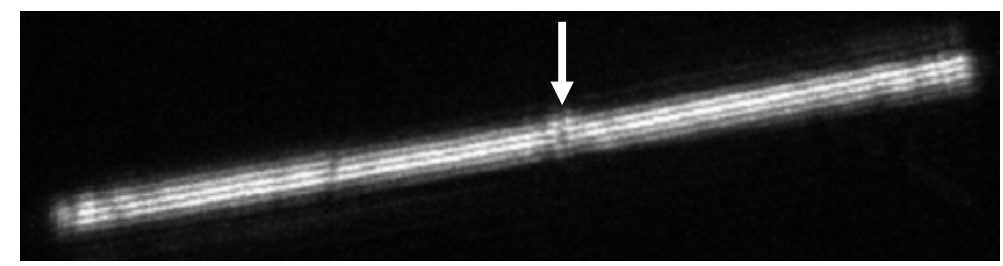

Fig 3 - Higher magnification of Fig 2 d showing the fringes more clearly and phase shifts existing at the intersections with other HOLZ lines, for example, at arrow. 